\title{
What is enumerated by subitization mechanisms?
}

\author{
GREG DAVIS \\ University of Cambridge, Cambridge, England \\ and \\ AMANDA HOLMES \\ Birkbeck College, London, England
}

\begin{abstract}
Subitization, the rapid enumeration of small numbers of objects, is often held to reflect a general ability to process three or four items simultaneously. However, questions as to which types of visual representations count as items for subitization have, thus far, received relatively little attention. In several new experiments, we instructed participants to enumerate three or four colored regions in a display. In some trials, all of these regions belonged to separate visual objects, whereas in others, two or more belonged to the same object(s). We found far slower enumeration in the latter case than in the former case, a result that cannot be attributed to Stroop interference from the number of attended objects in a display. We conclude that subitization mechanisms cannot operate on small fragments of shape and color alone, even when such fragments are easily individuated from one another. Rather, such mechanisms are compelled to operate on object representations.
\end{abstract}

Human observers can enumerate up to four visual items rapidly and accurately with little loss in performance, relative to enumerating only one or two items $(\approx 40 \mathrm{msec} /$ item; see, e.g., Trick \& Pylyshyn, 1994). However, when five or more items must be enumerated, performance starts to deteriorate, responses becoming markedly slower $(\approx 200$ $240 \mathrm{msec} / \mathrm{item}$ ) and less accurate with each extra item that must be enumerated (e.g., Trick \& Enns, 1997). The ability to enumerate three or four items as efficiently as one or two is termed subitization and is held to reflect parallel, preattentive processing of items (see, e.g., Pylyshyn, 2001). In contrast, serial, attentional shifts are thought to be necessary when larger numbers of items are counted. One popular approach in the literature holds that our ability to subitize reflects a limited capacity mechanism that can store up to four items in parallel. Each slot in this store is referred to as a FINST (finger of instantiation), and only when the four-item capacity is exceeded (when five or more items must be counted) are serial attentional shifts required to process all the relevant items. Other plausible explanations, which do not suppose a fixed number of discrete slots, have also been advanced to account for the same data (e.g., van Oeffelen \& Vos, 1982; Wender \& Rothkegel, 2000). However, these contrasting approaches have in common the view that subitization reflects an abil-

This work was funded by an E.P.S.R.C. and Ministry of Defence (U.K.) grant. Our thanks to Tom Carr, Lana Trick, and Jacob WaldmanKhaira for comments on an earlier draft. Correspondence concerning this article should be addressed to G. Davis, Department of Experimental Psychology, University of Cambridge, Downing Street, Cambridge CB2 3EB, England (e-mail: gjd1000@cam.ac.uk). ity of human observers to enumerate three or four items simultaneously.

Whereas many previous studies have examined whether subitization reflects qualitatively different types of processing from serial counting of larger numbers of objects, relatively little attention has been paid to which visual representations count as items for the purposes of enumeration. Pylyshyn and colleagues have taken a neutral position on this issue, preferring to refer to units in their tasks as items; these may be objects, small clusters of features, or individual features (see, e.g., Burkell \& Pylyshyn, 1997). To clarify, we use the term individual features to refer to visual primitives such as color, orientation, and texture, and the term feature clusters to refer to a bound set of individual features appearing at the same location. An example of a feature cluster would be a red vertical object, where orientation, shape, and color information all appear at the same location. Multiple feature clusters can also form parts of larger object representations - for example, when two tilted, colored objects form a single $\mathrm{V}$ shape. In the present experiments, we assessed whether individual features, feature clusters, or object representations form the primary units or items enumerated by subitization mechanisms. Alternatively, two or more of these candidate representations may be enumerated in parallel or compete for control of subitization mechanisms.

In our experiments, two basic predictions were contrasted: (1) that objects are the primary units of subitization or (2) that either features or feature clusters form these units. The logical basis of most of our experiments was as follows. If features or clusters of features, rather than objects, form the primary units of subitization, they should be enumerated with equal efficiency, irrespective of whether or not they belong to the same object. Con- 
versely, if object representations form the primary units of subitization, enumeration of features should be substantially slowed when two or more of the features belong to the same object. In such a case, top-down, attentional processing may be required in order to serially count the two separate features belonging to a single object (which would be counted as only one unit by subitization mechanisms). In addition, one study reported here tested the reverse conditions: whether individual features present in the display can affect enumeration of the target objects there. If objects are the primary or sole units of subitization, they should be enumerated irrespective of the features they comprise. However, if features or feature clusters are the primary units of subitization, we should expect effects of the number of salient features in a display upon enumeration of target objects. One further possible pattern of results is that we might find evidence of interference in both these types of task. In this case, we would conclude that both features and objects may form the units of subitization, a scenario we will discuss later.

Several previous studies of enumeration are particularly relevant to this discussion. For example, Trick and Enns (1997) explored whether a range of different target items in visual search displays could be subitized or not. They found that (with some exceptions) when target items were easily distinguished from distractors, they could be subitized. That is, subitization arose when target features popped out from distractors, due to their being differentiated from the distractors in terms of one low-level feature (e.g., color or orientation), but did not arise when target features differed from distractors only in terms of feature combinations (and the target features did not pop out). The lack of subitization for conjunctions of target features that did not pop out may reflect either or both of the following: (1) The items of subitization may be individual features, rather than locally bound feature clusters or perceptual objects, and/or (2) there may be an inability to attend target features selectively, while ignoring nontarget features. In the present experiments, we examined the effects of object segmentation on enumeration of easily identified target features, where the individual effects of these two factors could be isolated.

Also relevant are experiments by Trick and Pylyshyn (1994). In these, the effects of difficulty of individuation on enumeration were examined: As items become progressively harder to distinguish, enumeration became substantially slower. In particular, concentric shapes were far harder to enumerate than other, randomly arranged shapes. One possible explanation for these findings is that the concentric shapes were treated as a single perceptual object and that the items of enumeration were wholeobject representations, rather than individual features. In such a case, several concentric or parallel shapes could be counted only as one item, irrespective of how many shapes there were, slowing responses. Two control studies suggested that these effects could not be accounted for in terms of lateral masking. However, for our purposes, it is uncertain whether the concentric shapes should be considered to be "objects" or local feature clusters (since their centers all appeared at the same location).

Note that if we were to find effects of object segmentation on enumeration of features/feature clusters, this could be described as an example of difficulty in individuation. If, when two or more feature clusters appear to form parts of a single object, they are less easily enumerated than two clusters belonging to separate objects, this would presumably be because the two features cannot be rapidly treated as two separate units for the purposes of enumeration. However, we would like to distinguish two potential sources of difficulty in individuation here: those due to the grouping of features into object representations (which we address here) and those due to other factors. Among the most notable of these potential "other factors" is proximity (when two clusters are sufficiently near each other or are centered at the same location, they may not be individuated; see, e.g., Trick \& Pylyshyn, 1994). In the present experiments, the effects of proximity are controlled for, leaving only the effects of object segmentation.

In the first experiment described here, we employed a task that, according to all previous literature, should be very simple: Observers were instructed to enumerate three or four colored rectangles that were entirely red (among green nontarget features). This was a fairly simple task and within the normally accepted subitization range of three to four items. We then examined the effects of object segmentation on this task. On some trials, two target features could appear on the same object (a vertical or a horizontal object), whereas on other trials, all the features appeared on separate objects. If feature clusters (or individual features) are the primary units of enumeration, this manipulation should make no difference. If, on the other hand, objects form the primary units of enumeration, we might expect slower performance in the former case. This prediction runs in the direction opposite to that of the expected effects of visual attention, which often yield shorter reaction times (RTs) when features appear on the same object, so any such effects are unlikely to be due simply to attention (e.g., Duncan, 1984).

Figures $1 \mathrm{~A}$ and $1 \mathrm{~B}$ illustrate typical horizontal object and vertical object displays in Experiment 1, respectively. In Figure 1A, there are four target features (four red regions, indicated by light gray), and in Figure 1B, three target features that were to be enumerated are shown. There were always only three or four target features. All the remaining object ends were filled with green nontarget features (indicated by darker gray in Figures $1 \mathrm{~A}$ and 1B). All of the red (target) and green (nontarget) colored regions were faded gradually to the black color of the rest of the object in each case. This was to ensure that the target and nontarget features appeared to be part of the objects. Nonetheless, the target features were easily visible, popping out from the green nontarget features and black regions of the objects.

In order to manipulate object segmentation independently of distance, we organized the vertical or horizontal objects in each trial into three pairs of objects. These pairs 


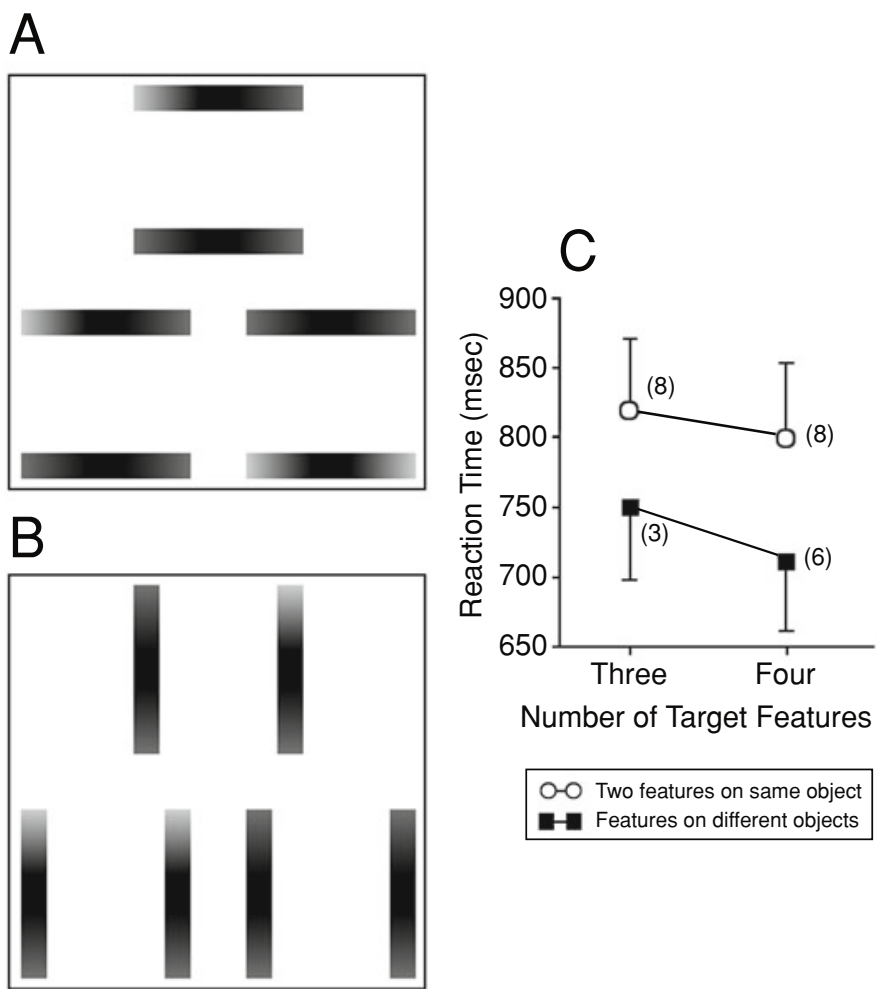

Figure 1. Displays and results from Experiment 1. (A) Display comprising horizontal bars and four red target items (indicated by light gray here) among other green nontargets (indicated by dark gray here). The remaining black middle sections of each bar in the figure were also black in the experiment. Note that two of the four target items fall on opposite ends of the same object. (B) Display comprising vertical bars and three target items. Two of the target items fall on adjacent ends of two neighboring objects within the bottom-left pair of objects (see the text for details). (C) Reaction time data (with percentages of errors in parentheses) for the conditions in Experiment 1. The left pair of data points indicates performance on three-target trials, and the right pair on four-target trials. The filled symbols graph performance on trials in which two of the target features appeared on adjacent ends of neighboring objects, and the open symbols indicate trials in which two of the target features appeared on opposite ends of a single object.

were arranged in a triangular formation, one in the center at the top, one on the bottom right, and one on the bottom left of the display (see Figures 1A and 1B). A maximum of two target features could appear within each pair of objects and appeared either (1) on opposite ends of the same object (as in Figure 1A) or (2) on adjacent ends of two different objects (as in Figure 1B). Note that each pair of objects forms two sides of an imaginary square, such that two target features are roughly equidistant from each other whether they are on the same object or on two separate objects. Hence, by comparing performance on trials in which the target features appeared on the same object with that on trials in which they were on adjacent ends of different objects, we could measure the effects of object segmentation independently of proximity effects (more stringent control for the effects of location were provided in a separate, control experiment, Experiment 2).

\section{EXPERIMENT 1 \\ Effects of Object Segmentation on Enumeration}

\section{Method}

Observers. Eight observers from the department subject panel were recruited. Six were female, and 2 were male; their ages ranged from 18 to 34 years, with a mean of 24 years. Each was paid $£ 7$.

Displays. The stimuli were presented on a Sony 17 -in. screen with a Power Macintosh G3 computer running VScope experiment generator software (Enns \& Rensink, 1992). A given trial comprised only horizontal objects (Figure 1A) or vertical objects (Figure 1B). These figures (and those for the other experiments) are drawn to scale: The actual stimuli measured approximately $22 \mathrm{~cm}$ vertically 
and were viewed from a distance of approximately $50 \mathrm{~cm}$. From this dimension, all other stimulus dimensions can be calculated, given the scaled figure. The stimuli were presented until a response was made, when a feedback symbol $(+$ or -$)$ appeared centrally, subtending $0.5^{\circ}$ of visual angle.

Procedure. The observers performed 10 blocks of 60 trials each, the first 4 of which were excluded as practice. Trials comprising vertical objects were randomly intermixed with those comprising horizontal objects, as were trials comprising four target features versus those with three target features. All possible trial types were equiprobable. Within each pair of objects (three pairs in each display in a triangular formation), only two target features could appear, and they could appear only at opposite ends of the same object or at adjacent ends of different objects within the pair. Other than this restriction, the features were equally likely to appear anywhere in the display. In the primary analysis, performance was compared on trials in which two of the three or four target features appeared on a single object with performance on trials in which two features appeared on neighboring objects within a single pair. For the reasons described above, spatial factors should be equivalent in the two cases. Additional analyses were performed for RTs and error rates on rare four-target trials in which all four target features appeared within two pairs of objects (about $10 \%$ of the trials). Performance on two types of trials was compared: first, trials in which two of the target features appeared on opposite ends of one object and two appeared on opposite ends of another object (such that four target features appeared on only two objects); second, trials in which two features appeared on adjacent ends of neighboring objects in one pair of objects and the other two features appeared on adjacent ends of neighboring objects within a second pair of objects (four target features on four separate objects).

\section{Results and Discussion}

Figure 1C graphs the interobserver means of the median RTs (with percentage error rates in parentheses), in the primary analysis, for three-target (left pair of data points) and four-target (right pair of data points) trials, respectively. The filled rectangular symbols indicate performance on trials in which two of the target features appeared on adjacent ends of neighboring objects, and the open circular symbols indicate trials in which two of the target features appeared on opposite ends of a single object. RTs were numerically longer and error rates were higher for conditions in which two of the features pertained to the same object. These effects were confirmed in a two-way within-observers ANOVA, with factors of number of target features (four vs. three) and object segmentation (two target features on the same object vs. different objects within a pair). The analysis yielded a significant main effect of four versus three target features $[F(1,7)=$ 7.96, $p=.026]$ : Four target features were enumerated more quickly than three, suggesting that target items were subitized (enumerated rapidly/in parallel) in our displays, rather than counted slowly and serially. More important for our present purposes, however, there was also a highly significant main effect of object segmentation: Items were enumerated more slowly (some $79 \mathrm{msec}$, on average) on trials in which two of the three or four target features appeared at opposite ends of the same object than on trials in which corresponding pairs of features belonged to adjacent ends of two neighboring objects $[F(1,7)=20.48$, $p=.003]$. There was no interaction $[\mathrm{F}(1,7)=0.43$, n.s. $]$.
Similar analyses performed on the error rates also revealed a significant main effect of object segmentation $[F(1,7)=9.59, p=.017]$, with performance having the same pattern as that for the RT data.

This primary analysis demonstrated that even in this easy task, in which target features popped out from distractors, there was a substantial effect of object segmentation. These data provide evidence that individual feature clusters, the target features in the experiment, are not the only items of subitization. If individual feature clusters were the primary representations to count as items, they should have been subitized with equal rapidity, independently of whether they belonged to separate object representations or not. However, the substantial drop in speed and accuracy of enumeration when two target features belonged to the same object indicated that such was not the case. Rather, on this initial evidence, object representations seem to have been the items of enumeration.

This effect may have arisen for two primary reasons (other than the factors we controlled for in the subsequent experiments). First, it may simply be that objects are the items that are the primary (or sole) basis of subitization. In such a case, when all target features appear on separate objects, subitization should still arise rapidly: Simply enumerating the objects upon which target features appear can yield the correct response. However, when two of the target features appear on the same object, the number of target features now differs from the number of objects upon which they appear. If there are four targets, they will appear on three objects; three targets will appear on two objects. If subitization mechanisms provide instant enumeration of objects, this does not provide a reliable means of enumerating target features (the task in our experiment). The second possible explanation holds that both objects and features are items of enumeration and that, when trying to enumerate objects, observers suffer Stroop-like interference from the number of objects on which the target features appear. Hence, when four targets are presented and two of them appear on the same object, three objects have targets on them. In this situation, the correct response (counting the number of target features) is four, but if enumeration mechanisms simultaneously count the number of objects, this would generate the response three, slowing the response to the number of features.

We shall discuss the latter, Stroop interference possibility and test it more directly in Experiment 5. However, a comparison of various conditions in Experiment 1 already provides some initial evidence against it. There is ample evidence that numerical Stroop interference becomes greater, the closer the interfering number is (numerically) to the correct response number (the semantic distance effect; see, e.g., Pavese \& Umiltà, 1998). Therefore, if a substantial proportion of the object segmentation effect in Experiment 1 was due to Stroop interference, this should lead to the following prediction for the four-target displays. When two of the four target features appeared on the same object, such that four target features appeared on three objects, Stroop interference should be maximal 
(since the number of objects comprising target features [3] was numerically closest to the number of targets [4]). However, when the four target features appeared on only two objects (two targets on one object, two on another), any Stroop interference should be measurably decreased. Accordingly, if the effect of object segmentation on trials in which all four features appeared on only two objects is much less than that for trials in which the four features appeared on three objects (as in our primary analysis), this would indicate that the effects of object segmentation in our experiment were due partially to Stroop interference.

However, such was not the case. In a secondary analysis (as has been described in the Method section), RTs and error rates were compared for trials in which four target features appeared on two objects (in different pairs of objects) with those for trials in which they appeared on adjacent ends of two pairs of neighboring objects. The former conditions showed comparable slowing ( $75 \mathrm{msec}$ ), relative to that observed in our primary analysis. This effect was significant in a within-observers ANOVA with a single factor of object segmentation $[F(1,7)=7.34, p=$ .03]. A similar analysis of error rates yielded a nonsignificant $[F(1,7)=3.5$, n.s. $]$ effect in the same direction as the RT result ( $8 \%$ vs. $3 \%)$.

We therefore suggest that the difficulty in enumerating target features when one or more belong to the same object arose because individual target features (and the local color-shape feature clusters they belonged to) cannot be subitized independently of the objects to which they belong. That is, neither features (color, texture, or orientation) nor feature clusters (bundles of features, all arising at the same location) are the units enumerated by subitization mechanisms. Rather, object representations seem to form the primary items enumerated by subitization mechanisms and seem to override any enumeration of individual target features/clusters.

However, two further considerations may threaten the conclusions in this experiment. First, the distance between target features appearing at opposite ends of the same object versus between target features at adjacent ends of neighboring objects was only approximately equated. The effect of object segmentation in Experiment 1 may, therefore, have reflected the slightly greater distance between target features in the latter case. Second and more likely, when two targets appeared on the same object, they may have been bar blurred into a single red bar, due to color assimilation. In two further experiments, we provided compelling evidence for both of these possibilities. First, in Experiment 2, we introduced gaps into the objects (see Figures 2A and 2B for typical displays). This manipulation retained the precise spatial positions of target and nontarget features in Experiment 1 but weakened the tendency to perceive any target features as belonging to the same perceptual object. If spatial separation of target features had played a major role in our findings in Experiment 1, we should find the same effects in Experiment 2. If, on the other hand, those effects had arisen due to object segmentation, they should now be greatly weakened in the new experiment.

\section{EXPERIMENT 2 Control for Distance Between Features}

\section{Method}

Observers. Eight observers from the department subject panel were recruited. Six were female, and 2 were male; their ages ranged from 20 to 38 years, with a mean of 25 years. Each was paid $£ 7$.

Displays, Apparatus, and Procedure. All aspects of the experiment were identical to those in Experiment 1, with the exception of the gaps introduced between the ends of each "object."

\section{Results and Discussion}

Figure 2C graphs RTs (with error rates in parentheses) in Experiment 2 in the same format as that in Figure $1 \mathrm{C}$ for Experiment 1. Note that with the introduction of gaps into the objects, there now was no effect of object segmentation: Trials in which two features belonged to the same object were enumerated just as rapidly as when all the features appeared on separate objects. This result was confirmed in an ANOVA identical to the primary analysis in Experiment 1 (two factors of object segmentation and number of targets), which yielded no significant main effect $[F(1,7)=1.65$, n.s. $]$ or interactions $[F(1,7)=0.21$, n.s.] involving the object segmentation term. Similar analyses on error rates similarly yielded no such effects [main effect, $F(1,7)=0.43$, n.s.; interaction, $F(1,7)=$ 1.51, n.s.].

These results clearly suggested that the effect of object segmentation in Experiment 1 could not be attributed to any differences in the spatial separation of the features or to the directing of any graduated changes from black to red in the objects. All these were preserved in Experiment 2, where no effects of object segmentation were found. However, the addition of gaps into the objects in Experiment 2 introduced high contrast edges between target features where none had existed in Experiment 1, obliterating any tendency in Experiment 1 for two target features to be perceptually blurred into a single red bar. Accordingly, the effects of object segmentation in Experiment 1 might still be accounted for by blurring. To preclude any such possibility, we replicated Experiment 2, using precisely the same red, black, and green regions, but with the following alterations. All the items appeared now against a gray background. Crucially, moreover, the white, high-contrast gaps between the ends of each object were present just as they had been in Experiment 2, except that they now appeared to take the form of white circles against the gray background. Hence, any tendency (or lack of it) for the red color of two target features to blur into one across these gaps should be equivalent in the two experiments.

Figure 3 illustrates typical displays in this new experiment. First, the six white circles were presented against a gray background for $750 \mathrm{msec}$ (as in Figure 3A for horizontal bar trials; not illustrated for vertical bar trials). The objects (with accompanying nontarget green features and target red features) were then added (see Figures 3B and $3 \mathrm{C}$ ). Note that the target, nontarget, and black regions of the objects were all physically the same as those in Experiment 2 (other than a few pixels where the black regions 


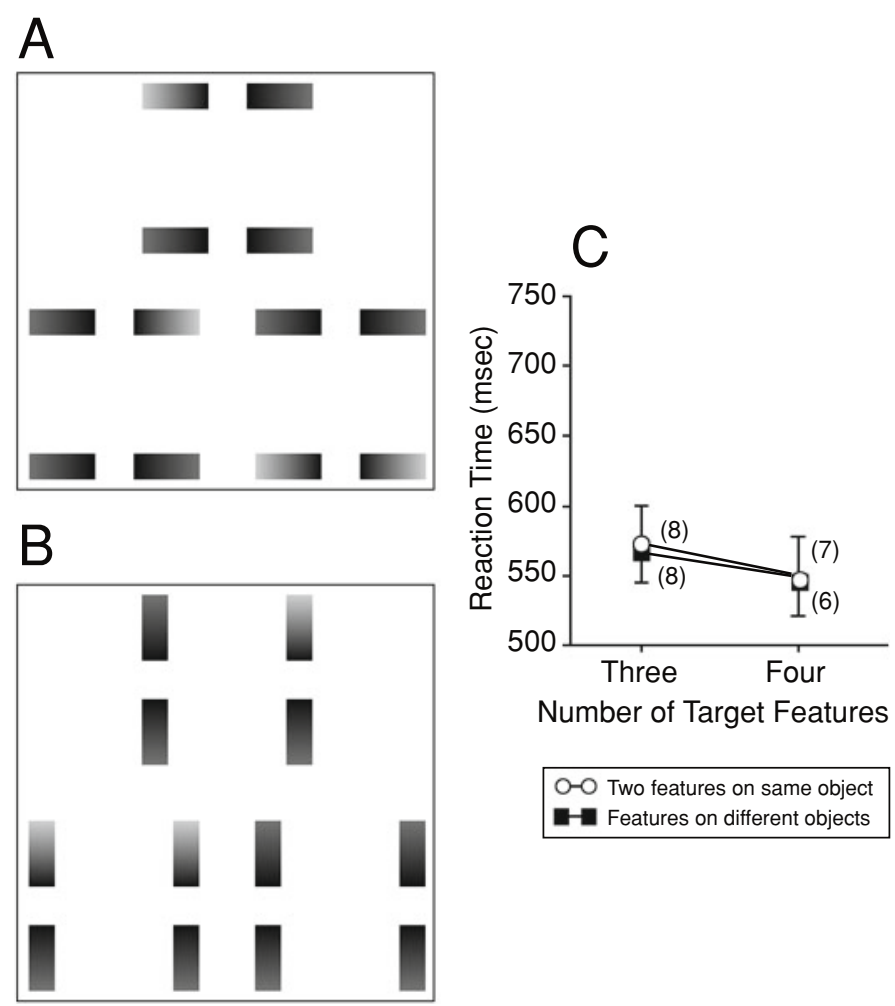

Figure 2. Displays and results from Experiment 2. (A) Display corresponding to the horizontal bar displays in Experiment 1 (but now with gaps introduced) and four red target items (indicated by light gray here) among other green nontargets (indicated by dark gray here). In the text, two target items at the bottom right are described as falling on opposite ends of the same "object," although with the white gaps introduced between the features, they no longer appeared as a single object. (B) Display comprising vertical bars and three target items. Two of the target items fall on adjacent ends of two neighboring objects within the bottom-left pair of objects (see the text for details). (C) Reaction time data (with percentages of errors in parentheses) for the conditions in Experiment 2. The left pair of data points indicates performance on three-target trials, and the right pair on four-target trials. The filled symbols graph performance on trials in which two of the target features appeared on adjacent ends of neighboring objects, and the open symbols indicate trials in which two of the target features appeared on opposite ends of a single object.

abutted the circles in Experiment 3). However, whereas we found no object segmentation effect in Experiment 2, we did expect to find an effect in the present experiment. The presence of the white circles now ensured that pairs of physically separate segments in the new displays appeared to form partially occluded horizontal or vertical bars in a display (see Figures 3B and 3C, respectively), due to the action of amodal shape completion mechanisms. We therefore predicted that despite the physical similarity of the new objects to those in Experiment 2, we should now find a clear effect of object segmentation. When two of the target features in a display belonged to opposite ends of an object, we expected to find deterioration in performance (in RTs and error rates), relative to conditions in which corresponding features belonged to adjacent ends of two separate objects within a single pair of objects (three pairs in each display; see, e.g., Figure 3B).

\section{EXPERIMENT 3}

\section{Enumeration of Features Belonging} to Occluded Objects

\section{Method}

Observers. Seven observers from the department subject panel were recruited. Five were female, and 2 were male; their ages ranged from 20 to 26 years, with a mean of 23 years. Each was paid $£ 7$.

Displays, Apparatus, and Procedure. All aspects of the experiment were identical to those in Experiment 2, with the exception of the gray background, which caused the white gaps between black regions belonging to the same object to take the form of white 

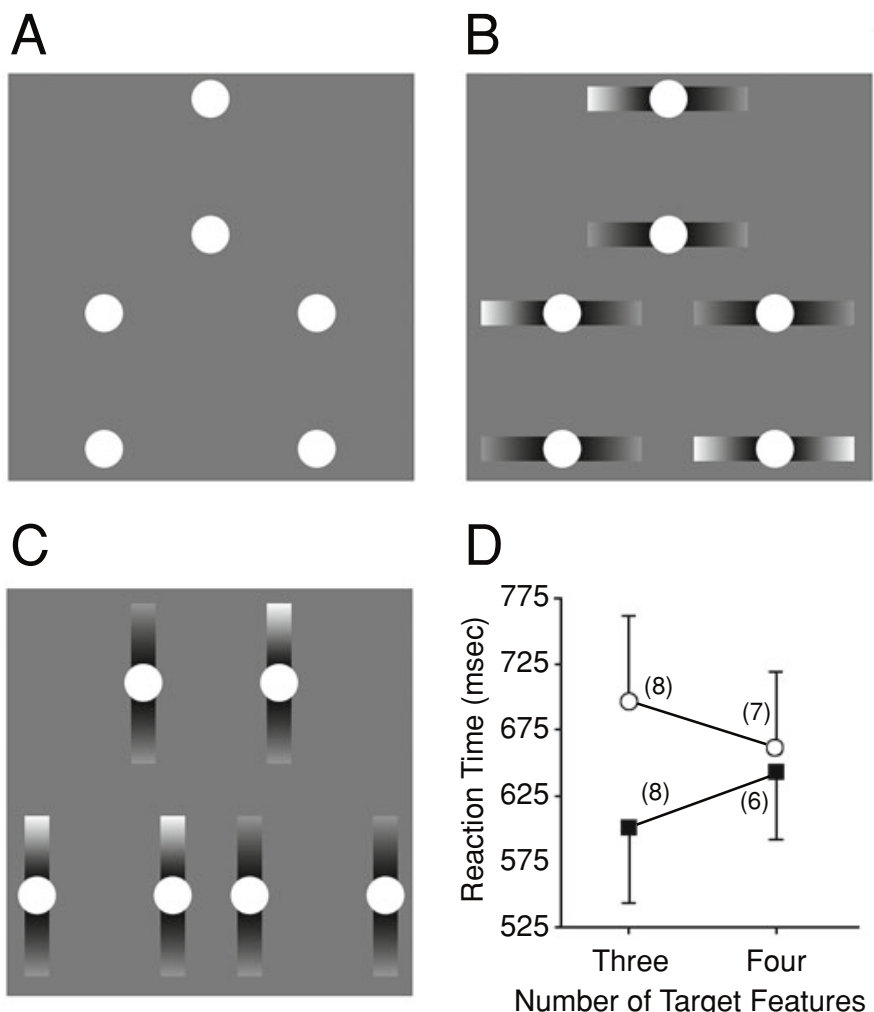

Number of Target Features

O-O Two features on same object

-1- Features on different objects

Figure 3. Displays and results from Experiment 3. (A) Display comprising a gray background and white circles, which preceded displays comprising horizontal bars (see, e.g., Figure 3B). In displays preceding vertical bar displays, the white circles were differently arranged so as to coincide with the white circles in those displays. (B) Typical horizontal bars display in Experiment 3 with four red target items (indicated by light gray) among other green nontargets (indicated by dark gray). Note that all of the black and colored (indicated by gray) regions of the objects were the same as those in Experiment 2, as were the white gaps between features belonging to the same object. However, the gray background in Experiment 3 meant that the white gaps now appeared as white circles. These circles elicited the perception of complete bars, even though the fragments making up these bars were the same as those in Experiment 2 (see the text). (C) Display comprising vertical bars and three target items. Two of the target items fall on adjacent ends of two neighboring objects within the bottom-left pair of objects. (D) Reaction time data (with percentages of errors in parentheses) for the conditions in Experiment 3. The left pair of data points indicates performance on three-target trials, and the right pair on four-target trials. The filled symbols graph performance on trials in which two of the target features appeared on adjacent ends of neighboring objects, and the open symbols indicate trials in which two of the target features appeared on opposite ends of a single object.

circles. The background and circles were presented for $750 \mathrm{msec}$ before the rest of the display in each trial. These circles elicited amodal completion of the black regions to form complete, partially occluded vertical or horizontal bars (see Figures 3B and 3C). Note that, also as in Experiment 3, the arrangement of the white circles was different according to whether vertical or horizontal bars were to be presented.

\section{Results and Discussion}

Figure 3D graphs the median RTs (with percentages of errors in parentheses), in the primary analysis, for threetarget (left pair of data points) and four-target (right pair of data points) trials, respectively. The open circular symbols indicate performance on trials in which two of the target 
features appeared on adjacent ends of neighboring objects, and the filled rectangular symbols indicate performance on trials in which two of the target features appeared on opposite ends of a single object. RTs were numerically longer (by $63 \mathrm{msec}$ ) and error rates were higher (by 4\%) for conditions in which two of the features pertained to the same object. These effects were confirmed in twoway within-observers ANOVAs, with factors of number of target features (four vs. three) and object segmentation (two target features on same object vs. different objects within a pair of objects). The analysis yielded no main effect of four versus three target features $[F(1,7)=0.37$, n.s.]: Four target features were enumerated as quickly as three, suggesting, as in the previous experiments, that our task had measured the action of subitization, rather than that of slow-counting mechanisms. As in Experiment 1, there was also a highly significant main effect of object segmentation: Trials in which two of the three or four target features appeared at opposite ends of the same object were enumerated more slowly than trials in which corresponding pairs of features belonged to adjacent ends of two neighboring objects $[F(1,6)=24.97, p=.003]$. There was an interaction of these two factors $[F(1,6)=$ $31.22, p=.001]$, indicating that the effect occurred primarily on the four target feature trials. Similar analyses performed on the error rates also revealed a significant main effect of object segmentation $[F(1,6)=13.98, p=$ $.01]$, with performance having the same pattern as that for the RT data. No effect of the number of targets or any interaction was found (both $F_{\mathrm{s}}<1.63$, n.s.).

Secondary analyses (one-factor within-observers ANOVAs on RTs and errors identical to those carried out on the Experiment 1 data) of trials in which four target features belonged to only two objects (vs. to adjacent ends of two pairs of objects) revealed effects for RT [96 msec; $F(1,6)=10.95, p=.016]$ and error rate $[9 \% ; F(1,6)=$ $16.62, p=.007]$ that were at least as big as those in the primary analysis. This again suggested that Stroop interference played little role in these effects. These results provide clear confirmation of our conclusion in Experiment 1 that object segmentation can have a substantial disruptive effect on the enumeration of target features even when they are readily distinguishable (i.e., they pop out) from nontarget items. This finding provides the clearest support yet for our contention that neither features nor feature clusters provide the sole units of enumeration. Rather, object representations seem to override any enumeration based on features or feature clusters and may themselves form the sole units of enumeration.

However, whereas Experiments 1-3 provided evidence that objects, rather than features, are the units of enumeration, it is difficult to be absolutely sure whether the enumeration involved reflects fast-subitization or slowcounting processes. Normally, if subitization had been active, we would expect that RTs to four-target displays should be only $40-50 \mathrm{msec}$ longer than those for threeitem displays, whereas this figure should be $250 \mathrm{msec}$ if slow counting had arisen. On this basis, our results sug- gest subitization. However, an unusual feature of our experiments means that we cannot conclude that subitization was present with any certainty. Since there were only two alternatives (three targets or four targets), observers might, in principle, have adopted a strategy to speed their responses for four item displays. If they were to assume that four items must be present (without enumerating them explicitly) whenever more than three were present, this would mean that four-item displays could yield RTs that were only slightly longer than those for the three-item displays in the absence of enumeration of the four items.

To test whether this was the case, Experiment 4 replicated Experiment 2 but now included trials comprising two, three, four, or five targets. Note that on four-target displays, this would prevent a strategy of assuming that four targets were present whenever more than three were detected. Similarly for three-target trials, detecting that fewer than four items were present would not yield the correct answer (either two or three targets might be present). Accordingly, for the three- or four-target displays, we could be sure that precise enumeration had taken place. Under these conditions, we made the following predictions. If the performance in Experiments 1-3 reflected subitization, RTs for the enumerating of four-target items in the new experiment would be only $40 \mathrm{msec}$ longer than RTs for the enumerating of four-target displays, even though more precise enumeration of targets in each display was required to perform the new task. Conversely, if our displays yielded slow counting of items, four-target displays should yield RTs that are around $240 \mathrm{msec}$ longer than those for three-target displays.

Note that our predictions did not relate to two- or fivetarget displays. This is because these displays may not have required precise enumeration, as did the displays in our previous experiments, and may therefore have yielded end effects (Trick \& Pylyshyn, 1994). For example, once an observer had determined that there were more than four targets in a display, he or she could assume that five targets must be present, without having to enumerate precisely how many targets were present.

\section{EXPERIMENT 4 \\ Two-, Three-, Four-, or Five-Target Displays}

\section{Method}

Observers. Seven observers from the department subject panel were recruited. Six were female, and 1 was male; their ages ranged from 18 to 33 years, with a mean of 26 years. Each was paid $£ 7$.

Displays, Apparatus, and Procedure. All aspects of the experiment were identical to those in Experiment 2, except that the number of targets could vary between two and five. The observers indicated the number of targets by pressing one of four labeled keys on a computer keyboard.

\section{Results and Discussion}

Median RTs for two-, three-, four-, and five-target displays were submitted to a one-way ANOVA, which, as was expected, yielded a highly significant effect of the number of target items on RTs $[F(1,6)=18.32, p<.01]$. Displays 
comprising two targets and five targets yielded shorter RTs (630 and $772 \mathrm{msec}$, respectively) than did those comprising either three or four target items. This was presumably because those conditions did not demand precise enumeration, as was mentioned in the Results and Discussion section of Experiment 3. More important, however, was the relationship between RTs for three- and four-target displays. Four target displays yielded RTs that were only $28 \mathrm{msec}$ longer than those for three-target displays (829 and $801 \mathrm{msec}$, respectively). Although this difference was significant $[t(6)=2.8, p=.03]$, it was clearly within the range expected for subitization processes $(40 \mathrm{msec}$ per item) rather than that for slow counting ( $240 \mathrm{msec}$ per item). Similar analyses of error rates yielded no significant effect of the number of targets $[F(1,6)=2.15$, n.s.]. Four-item displays did not elicit significantly more errors than did three-item displays $[t(1,6)=1.2$, n.s.; $4.6 \%$ vs. $3.2 \%$, respectively].

Together, the results of Experiments 1-4 provide clear evidence of an object-segmentation effect upon subitization mechanisms. These effects are consistent with our conclusion that objects, rather than features or clusters of features, form the representational units that are enumerated by subitization mechanisms. However, our results might also be accounted for if one were to assume that both objects and features act as units for subitization mechanisms. On this alternative view, the effects we found of object segmentation on performance for enumerating the number of target features would reflect something similar to the Stroop explanation that we discussed earlier. Whenever two target features appeared on a single object, subitization mechanisms enumerating target features would yield an answer different from that provided by subitization mechanisms enumerating the number of objects on which they appeared. The incongruence of these two answers would then slow responses.

In Experiments 1-4, therefore, the alternative view makes the same predictions as does our suggestion that object representations form the primary units of subitization. However, when the task is to enumerate the number of objects in a display, the two approaches make different predictions. For example, if the task were to enumerate three or four black objects in a display, the alternative view (that both features and objects can be enumerated by subitization mechanisms) would predict significant effects of the number of salient red features on those bars, lengthening RT when the number of red features was different from the number of objects. In contrast, our own view, that only objects (not features) are enumerated by subitization mechanisms, would predict a complete absence of any such effect. We conclude that objects may be the sole representations enumerated by subitization, such that when objects must be enumerated, there will be no interference from the number of salient red features in the display.

Experiment 5 tested the predictions from these two approaches. The task was to count the number of partially occluded bars in a display, while ignoring the salient red color features that appeared on the objects. Any effects of the number of salient red features on performance would provide evidence for the alternative view, that both features and objects are enumerated by subitization mechanisms. In contrast, a complete absence of such an effect would provide evidence for our contention that objects alone, and not features, form the units of subitization.

\section{EXPERIMENT 5 Enumeration of Objects}

\section{Method}

Observers. Seven observers from the department subject panel were recruited. Three were female, and 4 were male; their ages ranged from 22 to 32 years, with a mean of 25 years. Each was paid $£ 7$.

Displays, Apparatus, and Procedure. All aspects of the experiment were identical to those in Experiment 3, with the following exceptions. Following a display that comprised six white circles (750 msec, as in Experiment 3; see Figure 3A), three or four partially occluded bars appeared and remained until a response was made. The bars could be vertical (Figure 4A) or horizontal (Figure 4B), and the observer's task was simply to enumerate these bars as quickly and accurately as possible, responding by pressing one of two keys on a computer keyboard. Independently of the number of objects in a given display, two, three, or four red patches (the same as the target features in Experiments 1-4) were presented, forming parts of the objects to be enumerated. The effect of the number of these features on enumeration of the target objects was then measured. Any such effects would provide evidence that both objects and features can form the units enumerated by subitization processes. In contrast, if no such effects arose, this would support our view that only objects, not features, are enumerated by subitization mechanisms.

\section{Results and Discussion}

Figure 4C graphs the median RTs (with percentages of errors in parentheses), in the primary analysis, for threetarget (left pair of data points) and four-target (right pair of data points) trials, respectively. The open circular symbols indicate performance on trials in which the number of salient red features was the same as the number of objects to be enumerated, and the filled rectangular symbols indicate trials in which the number of salient red features was different from the number of objects. It is clear from the graph that the congruence of the number of features versus the number of objects made very little difference. RT differences averaged $5 \mathrm{msec}$ in favor of trials in which the number of features and objects were the same, with percentage error rates lower, on average, where the number of features and number of objects were different.

The absence of any effects was confirmed in two-way within-observers ANOVAs, with factors of number of target objects (four vs. three) and congruence of the number of objects versus number of features (same vs. different). For RTs, this analysis yielded a main effect of four versus three target objects $[F(1,6)=17.26, p<.01]$ : Three target features were enumerated $22 \mathrm{msec}$ more quickly than four. However, more important for our purposes, there were no main effects or interactions involving the congruence term (both $F \mathrm{~s}<1$, n.s.). Identical analyses of error rates similarly revealed no main effect or interaction 

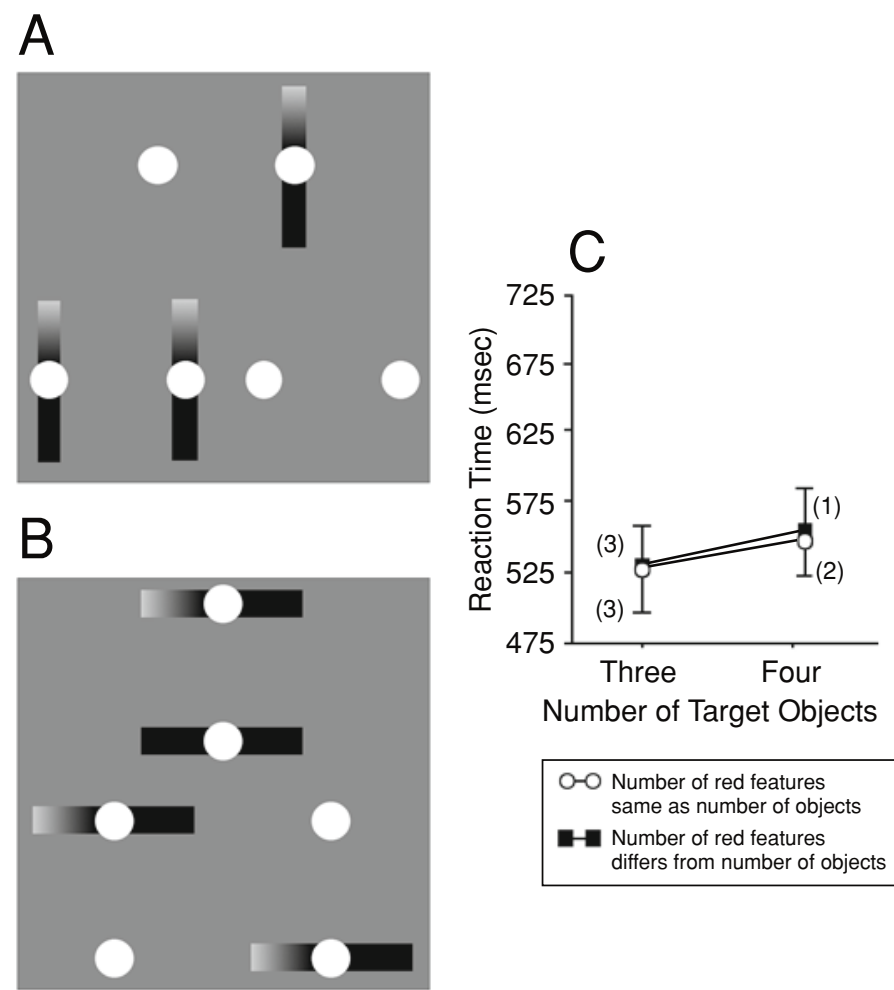

Figure 4. Displays and results from Experiment 5. (A) Typical vertical bars display with three target objects (partially occluded bars). In this case, there are three salient red features (indicated by light gray), which were irrelevant to the task, but which might be expected to affect performance. (B) Typical horizontal bars display with four target objects. There are again three salient red features (indicated by light gray), which were irrelevant to the task, but which might be expected to slow responses (see the text). (C) Reaction time data (with percentages of errors in parentheses) for the conditions in Experiment 5. The left pair of data points indicates performance on three-target trials, and the right pair on four-target trials. The filled symbols graph performance on trials in which the number of salient (but irrelevant) red features was different from the number of objects to be enumerated, and the open symbols indicate trials in which the number of salient red features was the same as the number of objects.

involving the congruence term (both $F \mathrm{~s}<1$, n.s.) and a nonsignificant trend for lower error rates on four-object than on three-object trials $[F(1,60=3.57, p=.108]$.

The complete absence of any effects of the number of target features in a display on performance in Experiment 5 supports our contention that only objects, not features, are enumerated by subitization mechanisms. These results are inconsistent with the view that both objects and features are enumerated by subitization mechanisms, which predicts an effect of similar proportion to that of object segmentation seen in our previous experiments (around 60-70 msec). Together, therefore, the results in Experiments 1-5 provide evidence that objects, not features, are the primary or sole units enumerated by subitization mechanisms. However, perhaps the most counterintuitive prediction generated by this conclusion was not tested by these experiments. If, as we suggest, only objects, not features, are enumerated by subitization mechanisms, we should find significant disruptive effects of object segmentation when two features appear on the same object, as we did in Experiments 1 and 3, even if the two color features are of different colors. If we were still to find such an effect, it would constitute the strongest evidence yet for our proposal. Under these new conditions, the two features on a single object should be very distinguishable from each other; hence, any remaining difficulty in enumerating these features (relative to when they belong to separate perceptual objects) must derive entirely from an inability to enumerate features belonging to the same perceptual object.

There were two basic types of trials to be compared in the new study. In object conditions, four circles were presented in a diamond formation (Figure 5A) for $500 \mathrm{msec}$ before the rest of the display was presented (Figure 5B). In each trial, four pairs of features were presented, forming four amodally completed bars. The observers were 
A

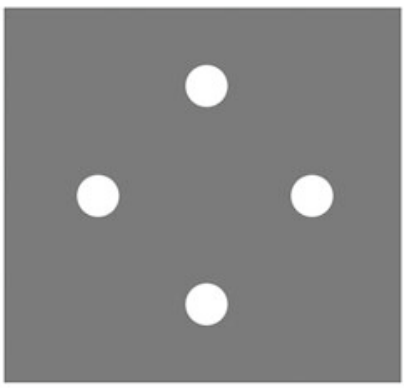

C

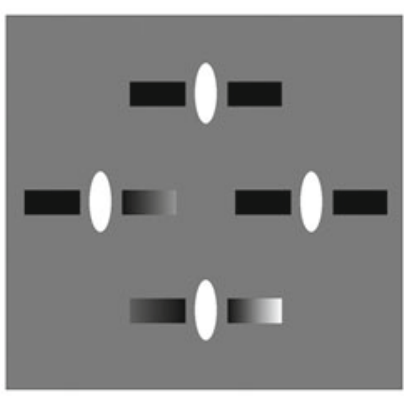

B

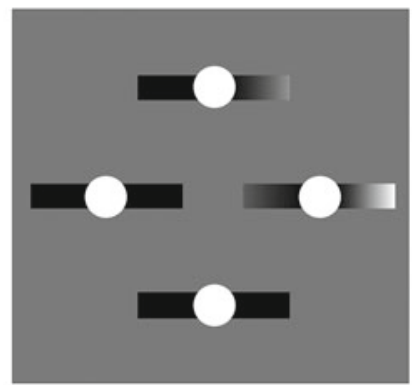

D

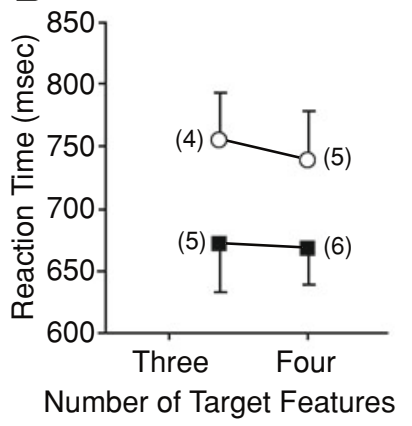

O-O Two features on same object

- Features on different objects

Figure 5. Displays and results from Experiment 6. (A) Display comprising a gray background and white circles, which preceded target displays in object condition trials. In separate condition trials, these circles were replaced by taller, thinner ellipses. In displays preceding vertical bar displays, the white circles were differently arranged so as to coincide with the white circles in those displays. (B) Typical object condition display in Experiment 4 with three colored target items (indicated by middle, light, and dark gray ends to the objects) among other black nontargets. The circles in these displays elicited the perception of complete bars, even though the fragments making up these bars were physically separate. (C) Separate condition display with three target items. Two of the target items fall within the bottom pair of objects and are physically identical to the right pair of features on a single object in panel B. Nonetheless, due to the presence of an ellipse (rather than a circle), these two features appear to belong to separate objects. (D) Reaction time data (with percentages of errors in parentheses) for the conditions in Experiment 2. The left pair of data points indicates performance on three-target trials, and the right pair on four-target trials. The filled symbols graph performance on trials in which two of the target features appeared on adjacent ends of neighboring objects, and the open symbols indicate trials in which two of the target features appeared on opposite ends of a single object.

instructed to ignore completely black nontarget segments and to enumerate the three or four colored target items. The items could be red, green, or blue, and each color faded to black gradually as it neared the abutting white circle (see Figures 5B and 5C). Crucially, when two target items appeared within a single object, they were never the same color. Accordingly, on any criterion, they were readily distinguishable from each other.

Performance for these object conditions was compared with that for the separate conditions. In the latter condi- tions, the colored and black regions of the displays were virtually identical to those in the object conditions (except for a few pixels where the black regions abutted the white circles in the object conditions). However, instead of circles, white ellipses were now presented such that each pair of segments (which would correspond to a single object in the object conditions) were now not perceived to form a single object.

If the apparent effects of object segmentation in the previous experiments were due to the fact that features 
(colored regions) are harder to enumerate when they belong to the same object than when they belong to separate objects, we should expect to find such effects in the new experiment. That is, there should be longer RTs and more errors in the object conditions, in which two target features appeared on the same object, than for corresponding trials in the separate conditions (in which two target features appeared in a single pair but were not joined into a single object). However, if the previous effects were due simply to lack of perceptual distinctiveness (which seems rather unlikely, since the target features were easy to distinguish from one another), we should expect to find no such effects here.

\section{EXPERIMENT 6 Enumeration of Differently Colored Features}

\begin{abstract}
Method
Observers. Eight observers from the department subject panel were recruited. Five were female, and 3 were male; their ages ranged from 19 to 31 years, with a mean of 24 years. Each was paid $£ 7$.

Displays, Apparatus, and Procedure. All aspects of the experiment were identical to those in Experiment 3, with the following exceptions. PsyScope, rather than VScope, software was employed. Four white shapes appeared in each trial (circles or ellipses; see Figure 5A for a circles display) for $500 \mathrm{msec}$ before presentation of the rest of the display. The main display (approximately $16 \mathrm{~cm}$ tall; drawn to scale in Figures 5A-5C) comprising the colored target regions and the black nontarget regions (Figures 5B and 5C) were then presented for $400 \mathrm{msec}$ before being replaced by a duplicate of the first display; this third display remained until a response was made. Colored regions (red, green, or blue) were now targets, and black regions were nontargets. In this new experiment, there were no four-target trials in which one target appeared on each object (or pair of object fragments in the separate conditions). This was to ensure that the observers could not respond to any trials on the basis of any absence of completely black objects in a display. Finally, object and separate conditions were blocked: Odd-numbered blocks were one condition, even-numbered blocks the other. Then the order of the blocks was counterbalanced across observers (half had object conditions in odd-numbered blocks; half had separate conditions in odd-number blocks).
\end{abstract}

\section{Results and Discussion}

Figure 5D graphs median RTs (with percentages of errors in parentheses) from trials in which two features appeared on the same object (in the object condition) or on two neighboring object fragments (in the separate condition). The left pair of data points is for three-target trials, and the right pair is for four-target trials. The open circular symbols indicate performance on object trials (as in Figure 5A), and the filled square symbols indicate performance for separate trials (as in Figure 5B). As we predicted, RTs were numerically longer and error rates were higher for conditions in which two of the features pertained to the same object (the object conditions) than when physically identical features belonged to separate objects (in the separate conditions). These effects were confirmed in two-way within-observers ANOVAs, with factors of number of target features (four vs. three) and object segmentation (object vs. separate displays). The analysis yielded no significant main effect of four versus three target features $[F(1,6)=1.59$, n.s.]: Four target features were not enumerated any more slowly than three, suggesting that serial counting had not arisen. As in Experiments 1 and 3, there was also a highly significant main effect of object segmentation: Items in trials in which two of the three or four target features appeared at opposite ends of the same object (the object conditions) were enumerated more slowly (some $77 \mathrm{msec}$, on average) than were those in trials in which corresponding pairs of features belonged to adjacent ends of two neighboring objects (in the separate conditions $[F(1,7)=28.82, p=$ $.001]$. There were no other main effects or interactions (all $F_{\mathrm{s}}<1.6$, n.s.). Similar analyses performed on the error rates also showed no significant main effects or interactions $\left(F_{\mathrm{s}}<1.6\right.$, n.s.), with performance having the same pattern as that for the RT data.

Secondary analyses (ANOVAs on RTs and errors identical to those carried out on the Experiments 1 and 3 data) of trials in which four target features belonged to only two objects (vs. to two pairs of segments in the separate conditions) revealed effects for RT [ $83 \mathrm{msec} ; F(1,7)=16.53$, $p=.005]$ comparable to those in the primary analysis but no significant effect for error rate $[F(1,7)=1.08$, n.s.]. This again suggested that Stroop interference played little role in these effects.

These results provide the most compelling demonstration yet that, when two target features belong to the same object, this engenders greater difficulty in enumerating them as two separate items than when they belong to separate objects. This quite substantial effect arises even when the targets are easily perceptually distinguishable from one another (due to their being different colors) and from nontargets.

\section{GENERAL DISCUSSION}

In the six new experiments described here, we have presented evidence that individual features and local feature clusters are not the units of enumeration. If they were, they should be enumerated equally rapidly, regardless of whether they belonged to the same object or to different objects. However, such is not the case: Whenever two of the features to be enumerated appear to form parts of the same perceptual object, they are enumerated much more slowly and less accurately than when they form parts of separate objects.

This effect of object segmentation on enumeration of features is a result neither of spatial separation of features (as we demonstrated in Experiment 2) nor of any tendency for target items to perceptually blur into one another (Experiment 3 ) nor of any difficulty in distinguishing them perceptually (Experiment 6). In addition, secondary analyses in the crucial experiments provided evidence that these effects were not due to Stroop interference but, rather, reflected an intrinsic difficulty in treating two features of the same object as separate units for the purpose of enumeration. Together, these results strongly support the view that 
object representations can override features or feature clusters as the items of enumeration, even against the observer's wishes (i.e., when the task is to ignore object information and count features, as it was here).

These results are also consistent with a second view, that both features and objects may form the units of enumeration. However, this view makes a further prediction not shared by our conclusion that objects are the sole units of subitization. When, in a reversal of the conditions in our other experiments, objects were to be counted and the effect of the number of salient features measured, this alternative view predicted a substantial effect of the number of features on performance. In contrast, our own view, that objects alone are subitized, not features or feature clusters, predicted no such effect. Experiment 5 showed absolutely no hint of this potential effect, providing support for our conclusion over the alternative view.

These results seem to relate closely to those found in the multiple object tracking paradigm. Scholl, Pylyshyn, and Feldman (2001) have recently investigated the effects of object segmentation on simultaneous tracking of three or four items, using this paradigm, and have found evidence that objects, rather than individual features or feature clusters, appear to be the basic elements involved in those tasks too. Similarly, Luck and Vogel (1997) have suggested that visual short-term memory also has objects as basic units, although this conclusion is controversial.

We would like to make two final points. First, although objects may be the units of enumeration, this does not always mean that the number of units we can enumerate at once is purely a function of the number of object representations. If the task were to enumerate object representations, the difficulty of the task might be dependent on the number of features those objects comprised, rather than on the number of objects per se. Further research should investigate this possibility. In addition, although we have used the term object here in order to contrast some types of representations (features and feature clusters) with others, it remains uncertain whether such a result would hold for all object representations. For example, it seems unlikely that spots would be harder to enumerate if they belonged to the same leopard, rather than to separate leopards. Rather, it may be that there is a very restricted set of "object" representations that can count as objects, and again, future research should address this issue.

\section{REFERENCES}

Burkell, J. A., \& Pylyshyn, Z. W. (1997). Searching through subsets: A test of the visual indexing hypothesis. Spatial Vision, 11, 225-258.

DunCAN, J. (1984). Selective attention and the organization of visual information. Journal of Experimental Psychology: General, 113, 501-517.

ENNS, J. T., \& RENSINK, R. A. (1992). VScope software and manual: Vision testing software for the Macintosh. Vancouver, BC: Micropsych software.

LUCK, S. J., \& Vogel, E. K. (1997). The capacity of visual working memory for features and conjunctions. Nature, 390, 279-281.

PAVESE, A., \& UMILtà, C. (1998). Symbolic distance between numerosity and identity modulates Stroop interference. Journal of Experimental Psychology: Human Perception \& Performance, 24, 1535-1545.

Pylyshyn, Z. W. (2001). Visual indexes, preconceptual objects, and situated vision. Cognition, 80, 127-158.

Scholl, B. J., Pylyshyn, Z. W., \& Feldman, J. (2001). What is a visual object? Evidence from target merging in multiple object tracking. Cognition, 80, 159-177.

Trick, L. M., \& ENNS, J. T. (1997). Measuring preattentive processes: When is pop-out not enough? Visual Cognition, 4, 163-198.

TRICK, L. M., \& Pylyshyn, Z. W. (1994). Why are small and large numbers enumerated differently? A limited-capacity preattentive stage in vision. Psychological Review, 101, 80-102.

VAn Oeffelen, M. P., \& Vos, P. G. (1982). A probabilistic model for the discrimination of visual number. Perception \& Psychophysics, 32, 163-170.

Wender, K. F., \& Rothkegel, R. (2000). Subitizing and its subprocesses. Psychological Research, 64, 81-92.

(Manuscript received July 7, 2003; revision accepted for publication February 14, 2005.) 